\title{
FRACTIONAL NOISES: DIFFUSIVE MODEL FOR CCD IMAGER BAND-PASS ACQUISITION CHAIN
}

\author{
FRANCIS LAVERNHE AND JOHANNES SOLHUSVIK
}

\begin{abstract}
Charge-Coupled Devices often deliver non standard noises, with a power spectrum proportional to a real power of $1 / \mathrm{f}$ over several decades (depending on biasing and temperature). On the other hand, CCD signal acquisition systems are frequently non stationary in their structure: the WienerKintchin theorem is not valid for evaluating noise power and signal to noise ratio. In this paper, the chain is seen as a continuous state-variable time-varying system with a fractional-noise input, modelled from a diffusive representation of fractional integrators.
\end{abstract}

RÉsumÉ. Le bruit des composants CCD est souvent non standard, à spectre de puissance proportionnel à une puissance réelle de $1 / \mathrm{f}$ sur plusieurs décades. Par ailleurs, les systèmes d'acquisition de signaux CCD sont fréquemment de structure non stationnaire: le théorème de Wiener-Kintchin n'est plus applicable pour l'évaluation de la puissance de bruit ou du rapport signal/bruit. Dans cet article, la chaine d'acquisition est considérée comme un système à coefficients variables, à variable d'état continu, attaqué par un bruit fractionnaire, modélisé à partir de la représentation diffusive d'un intégrateur fractionnaire.

\section{INTRODUCTION}

The sensitivity of CCD imagers is limited by the noise of the on-chip MOSFET amplifier (fig1) which generates both white noise and low-frequency noise with a power spectrum proportional to $1 / f^{\alpha}, 0.8 \leq \alpha \leq 2$ (fig2), depending on biasing, temperature and the fabrication process [1].

The function of a CCD signal acquisition system is to extract the pixel information from the output signal while suppressing the noise. The information, i.e. the amount of charge collected in a pixel, is represented by the voltage difference $(\Delta V)$ between the reference level after having reset the output node and the level after the charge injection from the pixel to the floating diffusion (see fig1). This technique is known as correlated double sampling (CDS).

Several ways for evaluating $\Delta V$ have been presented in the literature [2], [3]. When the output signal of the acquisition chain is stationary, standard tools in the frequency domain may be employed and the optimal solution, with respect to the signal to noise ratio, depends on the matching between the acquisition chain transfer function and the noise power spectral density (PSD). In opposite, other CDS methods are based on commutable filters whose both output and noise signals never reach the stationary rate [3], [4]. In that case, the mathematical representation of the system necessarily involves a dynamic state representation with $1 / f^{\alpha}$ input noise.

(C) Société de Mathématiques Appliquées et Industrielles. Typeset by LATEX.

F. Lavernhe: CIMI group, Electronic dept., ENSAE (Supaero), 31400 Toulouse Cedex, France. E-mail: lavernhe@supaero.fr.

J. Solhusvik: ABB corporate research, electronic systems dept., p.o. Box 90, N-1361 Billingstad, Norway. E-mail: jso@nocrc.abb.no. 


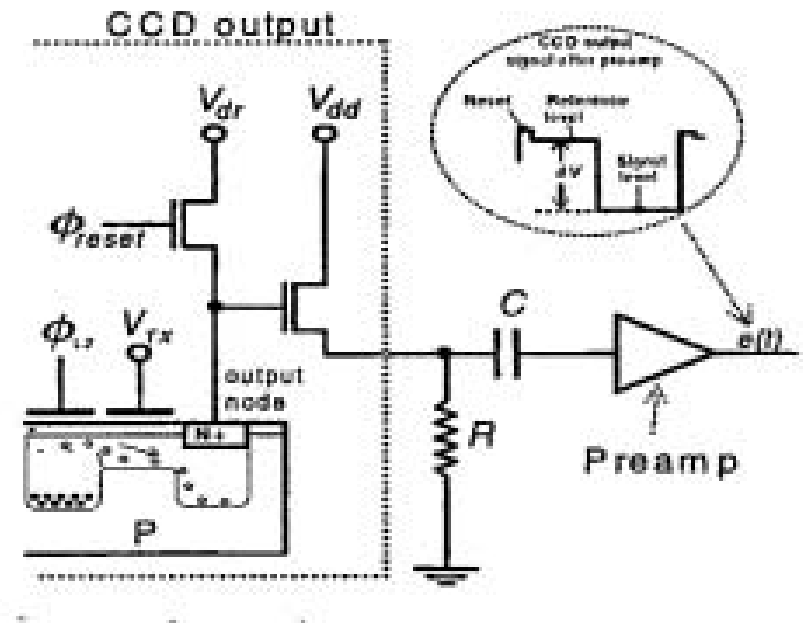

Figure 1. Shematic figure of a CCD output

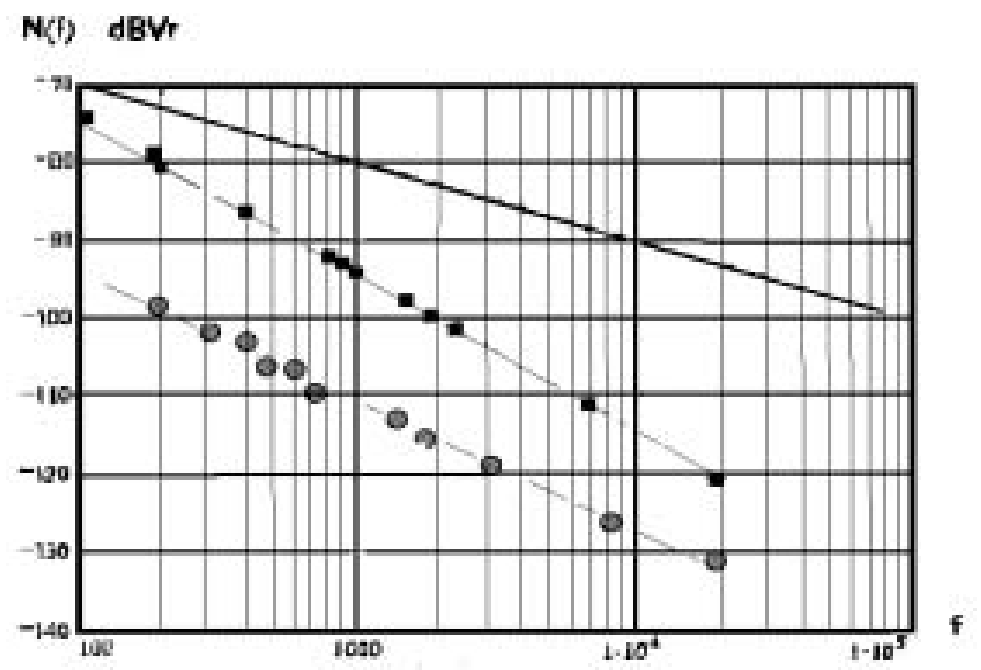

Figure 2. Output noise of a Thomson CCD detector type 7895M

The fractional $1 / f^{\alpha}$ noise $(0<\alpha<2)$, related to the fractional brownian motion [5], is difficult to manipulate under its usual formulation. Indeed, the time-convolution with a singular long-memory kernel leads to stochastic integrodifferential equations. The so-described stochastic process is not markovian: from the numerical simulation point of view, its entire past is to be memorized, which leads in practice to very prohibitive memory space and computation.

The approach presented in this paper is introduced in [6]; it was first used in [4] for the electronic treatment of the CCD noise. It is based on a markovian inputoutput representation of fractional noises, elaborated from an infinite-dimension stochastic differential equation. A rigorous and general mathematical frame is presented in [7]. 


\section{Preliminary notions}

We implicitly refer to an underlying probability space and the mathematical expectation of a real random variable $X$ is denoted by $E(X)$, the variance by $\operatorname{var}(X) ; t$ denotes the time variable and $f$ the frequency.

\subsection{The mathematical Model Under CONSIDERATion}

We consider the linear (stochastic) differential system:

$$
\frac{d x(t)}{d t}=A(t) x(t)+B(t) y(t), \quad x(0)=x_{0},
$$

with $y$ a scalar $1 / f^{\alpha}$ noise, $0 \leq \alpha \leq 2$, which means that the power spectral density $N_{y}(f)$ of $y$ is expressed as:

$$
N_{y}(f) \propto \frac{1}{|f|^{\alpha}}=\frac{1}{|f|^{2 \nu}}, \quad \alpha=2 \nu ;
$$

$y$ is linked to the standard white noise $w$ from an integration of non-integer order $\nu=\alpha / 2$. Note that in practice, the frequency domain of $\frac{1}{f^{\alpha}}$-noise covers several decades in CCD systems.

If $\alpha=0, y(t)$ is a white noise and (2.1) is written as:

$$
d x(t)=A(t) x(t) d t+B(t) d \beta(t),
$$

with $y=\frac{d \beta(t)}{d t}, \beta(t)$ a Wiener-Levy process or standard Brownian motion ${ }^{1}$ [8]. If $\alpha \neq 0, y(t)$ may be linked to a fractional brownian motion (FBM), the definition of which is recalled in section 2.3 .

\subsection{THE FRACTIONAL BROWNIAN MOTION}

The frequency-domain definition of the fractional noise is not sufficient when the system is not time-invariant. It is then necessary to consider a convenient timedomain definition. This last is based on fractional brownian motions. Given a constant $0<a<1$, the fractional brownian motion (FBM), with Hurst parameter $a$ and initial value $y_{0}$, may be defined for $t>0$ by :

$y_{f b m}(t)=y_{0}+\frac{(2 \pi)^{a+1 / 2}}{\Gamma\left(a+\frac{1}{2}\right)}\left\{\int_{0}^{t} \frac{d \beta(s)}{(t-s)^{\frac{1}{2}-a}}+\int_{-\infty}^{0}\left(\frac{1}{(t-s)^{\frac{1}{2}-a}}-\frac{1}{(-s)^{\frac{1}{2}-a}}\right) d \beta(s)\right\}$

This process has remarkable properties [5]. According to the definitions of the white noise and the fractional integral (of order $\nu>0$ ), denoted $I^{\nu}[9]$ :

$$
I^{\nu} h(t)=\frac{1}{\Gamma(\nu)} \int_{0}^{t} \frac{h(s)}{(t-s)^{1-\nu}} d s,
$$

$y_{f b m}(t)$ may be expressed under the form:

$$
y_{f b m}(t)=(2 \pi)^{\nu} I^{\nu} w(t)+\varsigma(t), \text { with } \nu=a+1 / 2,1 / 2<\nu<3 / 2 .
$$

The quantity $\varsigma(t)$ may be seen as "a corrective term" devoted to the property of stationary increments.

Remark: It may be shown that $I^{\nu} w(t)$ asymptotically behaves as $y_{f b m}(t)$, in then sense that:

1. $I^{\nu} w(t)$ possesses asymptoticaly stationary increments,

2. $\Delta_{\tau} y_{f b m}(t)-\Delta_{\tau} I^{\nu} w(t) \underset{t \rightarrow+\infty}{\stackrel{q . m}{\rightarrow}} 0$, with $\Delta_{\tau}$ the usual difference operator ${ }^{2}$.

\footnotetext{
${ }^{1}$ This process has independant increments, with the well-known properties: $E[w(t) . w(s)]=$ $\delta(t-s), E\left[(d \beta(t))^{2}\right]=d t$.

${ }^{2}$ This operator is defined by: $\Delta_{\tau} \varphi(t):=\varphi(t)-\varphi(t-\tau)$
} 
The FBM is the stationarized (in the increment sense) $\nu$-integration of a white noise process, with $1 / 2<\nu<3 / 2$. As usual in physics, we will call such a process, the "fractional noise of order $\nu ", 1 / 2<\nu<3 / 2$. For $0<\nu<1 / 2$, the fractional noise of order $\nu$ is the derivative of the FBM of parameter $a=\nu+1 / 2$. Such a process is then stationary if $\nu<1 / 2$, and stationary-increment if $\nu>1 / 2$; it behaves asymptotically as $I^{\nu} w(t)$. In the sequel, we only consider the case $\nu \geq 1 / 2$. The case $\nu<1 / 2$ may be tackled in a similar way, by considering, as in [6], the derivative of a FBM of parameter $a<1 / 2$.

\section{Diffusive markovian Representation of the Fractional noise}

This representation have its origin in the diffusive representations of fractional integrators introduced in [10] and successfully used in [11], [12]. It permits to translate the functional mapping : $z:=I^{\nu} u$ into the input-output diffusion equation with null initial condition:

$$
\left\{\begin{array}{l}
\frac{\partial Y(r, t)}{\partial t}=\frac{\partial^{2} Y(r, t)}{\partial r^{2}}+\delta(r) \cdot u(t), \quad Y(0, t)=0 \\
z(t):=\int_{-\infty}^{+\infty} m_{\nu}(r) Y(r, t) d r,
\end{array}\right.
$$

with $m_{\nu}(r)$ a convenient distribution. From Fourier transform with respect to $r$, and introducing a non null initial condition in order to warrant the stationarity of the process, we consider in the same way:

DeFinition 3.1. The Diffusive stationary input-output representation is defined as:

$$
\left\{\begin{array}{l}
d y_{\xi}(t)=-\xi y_{\xi}(t) d t+d \beta(t), \quad y_{\xi}(0)=y_{\xi 0}, \quad \xi>0, \\
y(t):=\int_{0}^{\infty} y_{\xi}(t) \mu_{\nu}(\xi) d \xi
\end{array}\right.
$$

The fractional noise $y$ will be elaborated from $(3.2)$, with $\mu_{\nu}(\xi)$ a real measure defined on $\mathbf{R}^{*+}$ to be specified. $y_{\xi 0}$ is a centered gaussian random variable chosen to impose the stationarity on the process.

Theorem 3.2. For $\mu_{\nu}(\xi)=\frac{\sin (\pi \nu)}{\pi}\left(\frac{2 \pi}{\xi}\right)^{\nu}, 1 / 2<\nu<1$, if $y_{\xi 0}$ verifies $E\left[y_{\xi 0}\right]=0$ and $E\left[y_{\xi 0}{ }^{2}\right]=\frac{1}{2 \xi}$, then $y(t)$ is a $1 / f^{\alpha}$-noise, $\alpha=2 \nu$.

The proof is a direct consequence of lemma $(4,5,6,7)$ in section 6 .

\section{Application to FRACTIONAL STOCHASTIC DifFERENTIAL LinEar} MODEL.

The aim of the sequel is to use the markovian representation of the fractional noise in the study of noise performances of a CCD chain acquisition. The introduction of the markovian model in (2.1), transforms this stochastic fractional differential equation into an ordynary one of infinite dimension. Classical stochastic computation may then be used. For convenience, we denote $(f \mid g)_{\xi}=\int_{0}^{\infty} f g d \xi$.

\subsection{A CONVENIENT REPRESENTATION}

Let us consider the linear equation (2.1), with $y$ a fractional noise of order $\nu$, $1 / 2<\nu<1$. From the above, this model may be rewritten as :

$$
\left\{\begin{array}{l}
d y_{\xi}(t)=-\xi y_{\xi}(t) d t+d \beta(t), \quad y_{\xi}(0)=y_{\xi 0}, \xi>0 \\
d x(t)=A(t) x(t) d t+B(t) .\left(\mu_{\nu} \mid y_{\xi}(t)\right), x(0)=x_{0}
\end{array}\right.
$$

with $\mu_{\nu}(\xi)=\frac{\sin (\pi \nu)}{\pi}\left(\frac{2 \pi}{\xi}\right)^{\nu}, 0<\nu<1$ and $E\left[y_{\xi 0}{ }^{2}\right]=\frac{1}{2 \xi}$. The following representation (convenient for the computation of moments) may also be considered, due to 
the linearity property:

$$
\left\{\begin{array}{l}
d y_{\xi}(t)=-\xi y_{\xi}(t) d t+d \beta(t), y_{\xi}(0)=y_{\xi 0}, \xi>0 \\
d x_{\xi}(t)=A(t) x_{\xi}(t) d t+B(t) y_{\xi}(t), x_{\xi}(0)=0, \xi>0 \\
d \bar{x}(t)=A(t) \bar{x}(t) d t, x(0)=x_{0} \\
x(t):=\left(\mu_{\nu} \mid x_{\xi}(t)\right)_{\xi \in \mathrm{R}^{*}+}+\bar{x}(t)
\end{array}\right.
$$

Denoting:

$$
\mathbf{X}:=\left(\begin{array}{c}
\mathbf{y} \\
\mathbf{x}
\end{array}\right):=\left(X_{\xi}\right)_{\xi \in \mathbf{R}^{*+}}:=\left(\begin{array}{c}
y_{\xi} \\
x_{\xi}
\end{array}\right)_{\xi \in \mathbf{R}^{*+}}, \mathbf{X}_{0}=\left(\begin{array}{c}
y_{\xi 0} \\
0
\end{array}\right)_{\xi \in \mathbf{R}^{*+}}
$$

and

$$
\begin{aligned}
\mathbf{A}: & =\left(A_{\xi}\right)_{\xi \in \mathbf{R}^{*+}}:=\left[\begin{array}{cc}
-\xi & 0 \\
B & A
\end{array}\right]_{\xi \in \mathbf{R}^{*+}}, \quad \mathbf{B}:=(B)_{\xi \in \mathbf{R}^{*+}}:=\left[\begin{array}{l}
1 \\
0
\end{array}\right]_{\xi \in \mathbf{R}^{*+}}, \\
\mathbf{C}: & =\left[\begin{array}{ll}
\mathbf{0} & M_{\nu}
\end{array}\right], \text { with } M_{\nu}:=\left(\mu_{\nu} \mid .\right)_{\xi \in \mathbf{R}^{*+}},
\end{aligned}
$$

the system may be rewritten under the tensor form:

$$
\left\{\begin{array}{l}
d \mathbf{X}=\mathbf{A X} d t+\mathbf{B} d t \\
d \bar{x}(t)=A(t) \bar{x}(t) d t, x(0)=x_{0} \\
x:=\mathbf{C X}+\bar{x}(t)
\end{array}\right.
$$

\subsection{The Correlation FUnction of the process $x$}

We suppose, without loss of generality, that $E\left[\mathbf{X}_{0}\right]=0$ and $x_{0}=0$, which implies that $E[\mathbf{X}(t)]=0$. The correlation function of $x: R_{x x}(t, s):=E\left[x(t) . x^{T}(s)\right]$, is obtained from the $\mathbf{X}$ one, defined by :

$$
R_{x_{\xi} x_{\eta}}(t, s):=E\left[x_{\xi}(t) . x_{\eta}^{T}(s)\right], \xi, \eta, t, s>0
$$

The correlation tensor $\left\{R_{x_{\xi} x_{\eta}}(t, s)\right\}$ is the solution of the set of equations [6]:

$$
\left\{\begin{array}{l}
\frac{d}{d s} R_{x_{\xi} x_{\eta}}(s, s)=A_{\xi}(s) \cdot R_{x_{\xi} x_{\eta}}(s, s)+R_{x_{\xi} x_{\eta}}(s, s) \cdot A_{\eta}^{T}(s)+B \cdot B^{T} \\
R(0,0)=\left[\begin{array}{cc}
\frac{1}{\xi+\eta} & 0 \\
0 & 0
\end{array}\right] \\
\frac{\partial}{\partial t} R_{x_{\xi} x_{\eta}}(t, s)=A_{\xi}(t) \cdot R_{x_{\xi} x_{\eta}}(t, s),
\end{array}\right.
$$

and the correlation function $R_{x x}(t, s)$ is given by :

$$
R_{x x}(t, s)=\left(\mu_{\nu} \otimes \mu_{\nu} \mid R_{x_{\xi} x_{\eta}}(t, s)\right)
$$

The properties (4.5) and (4.6) are obvious (see ([6]) for the general case) and will be sued in the next section for CCD application. Due to the linearity of the system (4.2), and using (8.10), (4.6) may be rewritten under the more convenient form if only the computation of the moments of the output signal is considered :

$$
R_{x x}(t, s)=\left(\varphi_{\nu}(\xi) \mid R_{x_{\xi} x_{\xi}}(t, s)\right), \quad \varphi_{\nu}(\xi)=4\left(\frac{2 \pi}{\xi}\right)^{2 \nu-1} \sin (\pi \nu)
$$

Details are given in the particular case of the CCD acquisition chain in the next section. 


\section{Application to commutable pass-Band filter for CCD signal ACQUISITION}

The acquisition chain is a commutable band-pass filter whose function is the removal of both low-frequency excess noise (or fractional noise) and high-frequency noise in order to maximize the signal to noise ratio. When the output signal is the reference level (fig 1) the time constants are made very small for resetting the system and memorize the reference level. In the second part of the lecture sequence, the time constants are chosen in order to obtain the maximum output level at a desired date.

\subsection{THE MODEL}

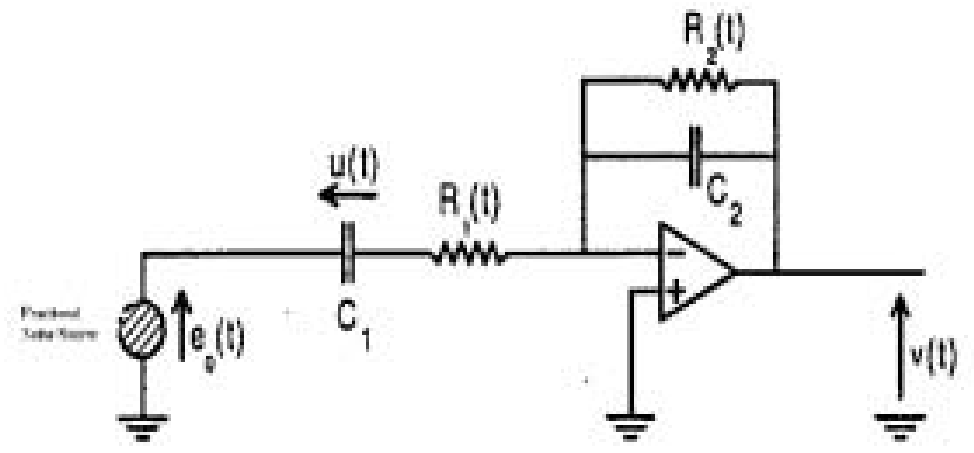

Figure 3. Variable band-pass filter

We consider, as in [4], the following state-representation of the commuted bandpass filter described in figure 3 , with fractional noise $y(t)$ input and output $s(t)$.

$$
\left\{\begin{array}{c}
d u=-a(t) u(t) d t+a(t) y(t) d t, \quad u(0)=0 \\
d v=-b(t) v(t) d t+c(t) u(t) d t-c(t) y(t) d t, v(0)=0
\end{array}\right.
$$

$a(t):=(R 1 . C 1)^{-1}, b(t):=(R 2 . C 2)^{-1}, c(t):=(R 1 . C 2)^{-1}$.

$R_{1}$ and $R_{2}$ are time-dependant resistors (using in practice JFETS as parallel switches). The function of this circuit is to reduce the noise bandwidth while measuring $\Delta V$. It allows to reset $C_{1}$ and $C_{2}$ at every period of the read sequence. Only the variance of $v(t)$ is of interest in this application. From (4.2), (5.1) is written :

$$
\left\{\begin{array}{l}
d y_{\xi}(t)=-\xi y_{\xi}(t) d t+d \beta(t) \\
d u_{\xi}=-a u_{\xi} d t+a y_{\xi} d t \\
d v_{\xi}=-b v_{\xi} d t+c u_{\xi} d t-c y_{\xi} d t \\
u(t)=\int_{0}^{\infty} u_{\xi}(t) \mu_{\nu}(\xi) d \xi \\
v(t)=\int_{0}^{\infty} v_{\xi}(t) \mu_{\nu}(\xi) d \xi .
\end{array}\right.
$$




\subsection{AnAlysis}

After convenient reorganization of (5.2), we obtain:

$$
d\left(\begin{array}{c}
y_{\xi} \\
u_{\xi} \\
v_{\xi}
\end{array}\right)=\left[\begin{array}{ccc}
-\xi & 0 & 0 \\
a & -a & 0 \\
-c & c & -b
\end{array}\right] \cdot\left(\begin{array}{c}
y_{\xi} \\
u_{\xi} \\
v_{\xi}
\end{array}\right) d t+\left[\begin{array}{c}
d \beta(t) \\
0 \\
0
\end{array}\right]
$$

rewritten as:

$$
d x_{\xi}=A_{\xi} x_{\xi} d t+[d \beta]
$$

where:

$$
x_{\xi}:=\left(\begin{array}{c}
y_{\xi} \\
u_{\xi} \\
v_{\xi}
\end{array}\right), A_{\xi}:=\left[\begin{array}{ccc}
\xi & 0 & 0 \\
a & -a & 0 \\
-c & c & -b
\end{array}\right],[d \beta]:=\left[\begin{array}{c}
d \beta(t) \\
0 \\
0
\end{array}\right]
$$

It is obvious that $E(v(t))=0$, and we denote $R_{w, z}\left(t, t^{\prime}\right):=E\left(v(t) . z^{T}\left(t^{\prime}\right)\right)$, so:

$$
\left\{\begin{array}{l}
R_{v v}\left(t, t^{\prime}\right):=E\left(v(t) v\left(t^{\prime}\right)\right)=\int_{0}^{\infty} \varphi_{\nu}(\xi) R_{v_{\xi} v_{\xi}}(t, v) d \xi \\
\text { with } \varphi_{\nu}(\xi):=4\left(\frac{2 \pi}{\xi}\right)^{2 \nu-1} \sin (\pi \nu) .
\end{array}\right.
$$

Using (5.4), and $t>t^{\prime}$,

$$
d R_{x_{\xi}, x_{\xi}}\left(t, t^{\prime}\right)=E\left(d_{t}\left(x_{\xi}(t) x_{\xi}\left(t^{\prime}\right)^{T}\right)\right)=E\left(A_{\xi} x_{\xi}(t) x_{\xi}\left(t^{\prime}\right)^{T} d t+[d \beta(t)] x_{\xi}\left(t^{\prime}\right)^{T}\right)
$$

where $E\left([d \beta(t)] . x_{\eta}\left(t^{\prime}\right)^{T}\right)=0$ because $\beta(t)$ is an independent increment process [14]. From linearity of (5.4), we then obtain:

$$
\frac{\partial}{\partial t} R_{x_{\xi} x_{\xi}}\left(t, t^{\prime}\right)=A_{\xi} R_{x_{\xi} x_{\xi}}\left(t, t^{\prime}\right)
$$

The evaluation of the tensor $\mathbf{R}_{x_{\xi} x_{\xi}}(t, t)$ is quite identical:

$$
d R_{x_{\xi}, x_{\xi}}\left(t, t^{\prime}\right)=E\left(d_{t} x_{\xi}(t) x_{\xi}(t)^{T}+x_{\xi}(t) d_{t} x_{\xi}(t)^{T}+d_{t} x_{\xi}(t) d_{t} x_{\xi}(t)^{T}\right),
$$

with (due to the classical rules of stochastic calculus) :

$$
E\left(d_{t} x_{\xi}(t) d_{t} x_{\xi}(t)^{T}\right)=E\left([d \beta(t)] \cdot[d \beta(t)]^{T}\right)=\left[\begin{array}{lll}
1 & 0 & 0 \\
0 & 0 & 0 \\
0 & 0 & 0
\end{array}\right] d t
$$

According to (8.2) and the stationarity of $y_{\xi}, E\left(y_{\xi}(t) y_{\xi}(t)\right)=\frac{1}{2 \xi}$; from the above and after convenient reorganization we obtain from (5.8), the infinite set of ordinary differential system (for each $\xi \in \mathbf{R}^{*+}$ ):

$$
\begin{aligned}
\frac{d}{d t}\left[\begin{array}{c}
R_{y_{\xi} u_{\xi}}(t, t) \\
R_{y_{\xi} u_{\xi}}(t, t) \\
R_{u_{\xi} u_{\xi}}(t, t) \\
R_{u_{\xi} v_{\xi}}(t, t) \\
R_{v_{\xi} v_{\xi}}(t, t)
\end{array}\right]=\left[\begin{array}{ccccc}
-\xi-a & 0 & 0 & 0 & 0 \\
c & -\xi-b & 0 & 0 & 0 \\
2 a & 0 & -2 c & 0 & 0 \\
-c & a & c & 0 & 0 \\
0 & -2 c & 0 & 2 c & -2 b
\end{array}\right] \cdot\left[\begin{array}{c}
R_{y_{\xi} u_{\xi}}(t, t) \\
R_{y_{\xi} v_{\xi}}(t, t) \\
R_{u_{\xi} u_{\xi}}(t, t) \\
R_{u_{\xi} v_{\xi}}(t, t) \\
R_{v_{\xi} v_{n \xi}}(t, t)
\end{array}\right] \\
+\left[\begin{array}{c}
\frac{a}{2 \xi} \\
\frac{-c}{2 \xi} \\
0 \\
0 \\
0
\end{array}\right], \xi>0 \text {, with null initial condition. }
\end{aligned}
$$


The variance of the output signal is finally:

$\left.\operatorname{var}(v):=R_{v \nu}(t, t)=4 \int_{0}^{\infty}(2 \pi / \xi)^{2 \nu-1} \sin (\pi \nu) R_{v_{\xi} v_{\xi}(t)} d \eta=\int_{0}^{\infty} \varphi_{\nu}(\xi) R_{v_{\xi} v_{\xi}(t)} d \xi 5.10\right)$ with $\varphi_{\nu}(\xi)$ defined in $(5.5)$.

We may obtain numerically a convergent evaluation of $\operatorname{var}(v)$ versus time by discretizing $\xi$. The proof of the convergence may be found in [7]. If we choose a finite set of positive numbers:

$$
0 \leq \xi_{0}<\xi_{1}<\ldots<\xi_{N-1}<\xi_{N},
$$

the reduction of $(5.9)$ to this set leads to the following approximation: $\operatorname{var}(v) \simeq$ $\sum_{i=0}^{N} \varphi_{\nu}\left(\xi_{i}\right) R_{v_{\xi_{i}} v_{\xi_{i}}(t)} \Delta \xi_{i}$. In practice, $N$ may be low (typically 10 ) which allows an implementation with very ordinary means.

This methodology has been implemented on a Thomson CCD detector (type $7895 \mathrm{M})$ whose output noise spectral density was proportional to $1 / f^{2}$ at room temperature (typically $295 \mathrm{~K}$ ) and $1 / f^{1.5}$ at $T=233 \mathrm{~K}\left(-40^{\circ} \mathrm{C}\right)$, in the aim to compare experimental data with the theoretical ones obtained from (5.10).

\section{Experimental versus theoretical results.}

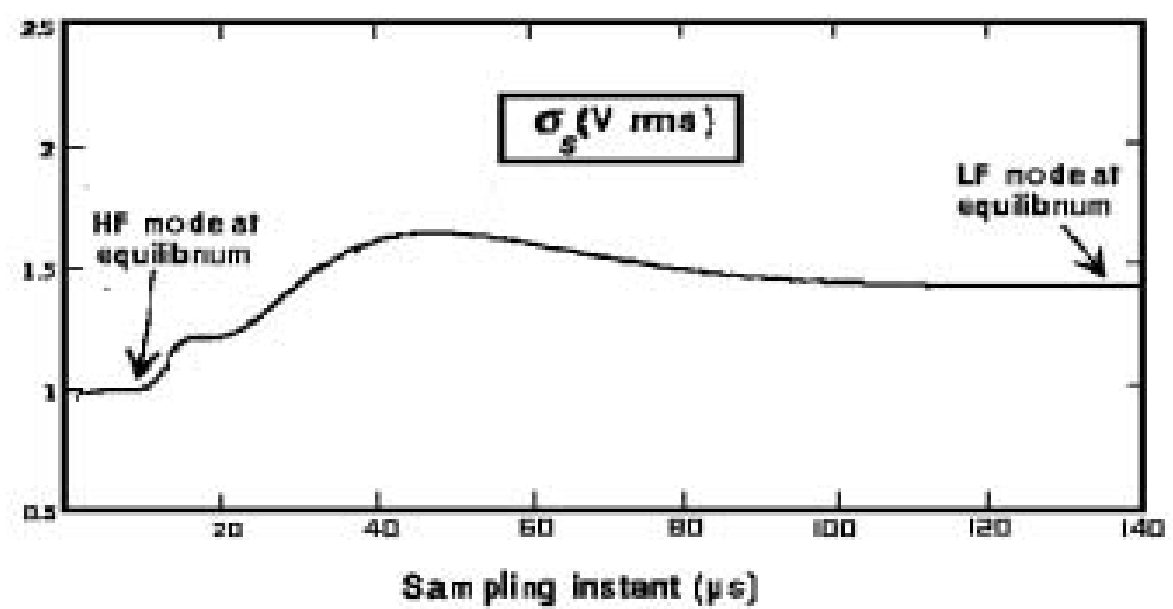

Figure 4. RMS output noise versus time with $1 / f$ input noise, $(\nu=0.5)$

Fig 4 shows the theoretical behavior of the square root $\sigma$ of the output variance for an input with $1 / f$ PSD. In fig 5 , both experimental points (dots) and theoretical (line) are included for $1 / f^{1.5}$ input noise. The experimental values of $\sigma$ are obtained by a direct measure on the output noise. The theoretical values come from the numerical resolution of the deterministic model (5.9) and 5.10). A good overall agreement is observed. The small discrepancies are due to our simplified model of the switches and operational amplifier during the transition. More technical details may be found in [13] and [4]

\section{Conclusion}

We have used a new markovian model of fractional noises. This approach allowed us to compare experimental and theoretical data of a band-pass filter used 


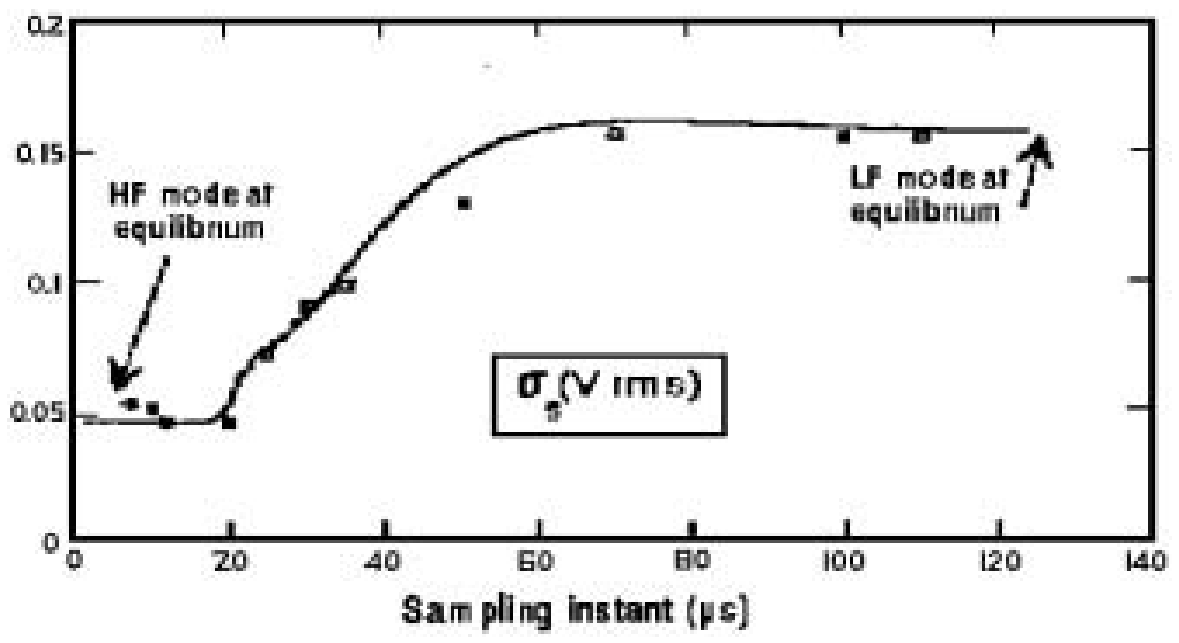

Figure 5. RMS output noise versus time with $1 / f^{1.5}$ input noise, $(\nu=0.75)$

in a CCD camera with a good agreement that confirms the validity of above approach. The model may also be used in Monte-Carlo simulations, for instance for the optimization of the signal to noise ratio. Remark that, in the hereditary model of the fractional noise, the integro-differential system is no more usable because of the computation and memory volume which is prohibitive (memorization of the past).

\section{REFERENCES}

[1] P.Centen, "CCD on-chip amplifiers: noise performance versus MOS transistor dimensions", IEEE Trans. electron devices, vol 38, pp. 1206-1216, may 1991.

[2] G.R.Hopkinson and D.H.Lumb, "Noise reduction techniques for CCD image sensors", J.Phys.E: Sci. Instrum., vol.15, 1982.

[3] Patent no: 9402577, Institut National de la Propriété Industrielle (INPI) 75800 Paris Cedex (France).

[4] J.Solhusvik, F.Lavernhe, G.Montseny and J.Farré," A new low noise signal acquisition method based on a commutable band-pass filter", IEEE Trans. on Circuits and Systems (II), vol. 44 no3, pp164-176, March 1997.

[5] B.B. Mandelbrot and J.W.Ness, "fractional brownian motion, fractional noises and applications", SIAM Rev., vol. 10, no. 4, pp 422-437, Oct 1968.

[6] F.Lavernhe, G.Montseny and J.Audounet, "Markovian diffusive representation of $1 / f^{\alpha}$ noises and application to fractional stochastic differential models, $L A A S$ report to be published in IEEE Trans. on Sig. Proc.

[7] P.Carmona, L.Coutin and G.Montseny, "A diffusive representation of fractional brownian motion with Hurst parameter less than 1/2", submitted to publication.

[8] L.Arnold, Stochastic differential equations (theory and applications), New York : Wiley, 1971.

[9] K.B. Oldam and J.Spanier, The fractional calculus- Theory and applications of differentiation and integration of arbitrary order. Academic Press, 1985.

[10] G.Montseny, J.Audounet,and B.Mbodje, "Optimal models of fractional integrators and application to systems with fading memory", IEEE SMC's Conference, Le Touquet (France), 1993.

[11] B.Mbodje and G.Montseny, "Boundary fractional derivative control of the wave equation", IEEE Trans. Aut. Cont, vol. 40,no. 2, pp 378-382, Feb 1995.

[12] G.Montseny, J.Audounet,and D.Matignon, "Fractional integrodifferential boundary control of the Euler-bernouilli beam", $36^{\text {th }}$ IEEE Conference on Decision and Control, Dec 10-12, 1997, San diego, California. pp 4973-4978. 
[13] Solhusvik J.,"Etude et conception de capteurs d'images à pixels actifs et de l'électronique de traitement associée en vue d'applications faible flux", Thèse de Doctorat en électroniqueoptronique, Ecole Nationale supérieure de l'aéronautique et de l'espace, Nov 19, 1996, Toulouse, France.

[14] Papoulis A.,"Probability, random variables and stochastic processes, New York, Mac GrawHill, 1991.

\section{ApPEndix}

LEMma 8.1. (stationarity of the process) The vector process $\left\{y_{\xi}(t)\right\}_{\xi>0}$ is centered, gaussian and WSS if and only if $\left\{y_{\xi}(t)\right\}_{\xi>0}$ is a centered gaussian random vector with covariance:

$$
E\left(y_{\xi 0} y_{\eta 0}\right)=\frac{1}{\xi+\eta}
$$

Proof. According to independant-increment property of $\beta$, the correlation function of $y_{\xi}$ is given, for any $s, t>0$, by:

$$
R_{\xi \xi}(t, s):=E\left[y_{\xi}(t) y_{\xi}(s)\right]=e^{-\xi(t+s)} E\left[y_{\xi 0}^{2}\right]+\frac{1}{2 \xi}\left(e^{-\xi|t-s|}-e^{-\xi(t+s)}\right)
$$

Thus a first necessary condition for $\left\{y_{\xi}\right\}_{\xi>0}$ to be stationary is:

$$
E\left[y_{\xi 0}{ }^{2}\right]=\frac{1}{2 \xi}
$$

which gives:

$$
R_{\xi \xi}(t, s)=\frac{1}{2 \xi} e^{-\xi|t-s|}
$$

Similarly, the asymptotic intercorrelation function of $\left\{y_{\xi}(t)\right\}_{\xi>0}$ is in the same way and after simplification given by:

$$
R_{\xi \eta}(t, s):=E\left[y_{\xi}(t), y_{\eta}(s)\right]=\left\{\begin{array}{cl}
\frac{e^{-\eta(s-t)}}{\xi+\eta} & \text { if } s>t \\
\frac{e^{-\xi(t-s)}}{\xi+\eta} & \text { if } s<t \\
\frac{1}{\xi+\eta} \text { if } s=t
\end{array}\right.
$$

we then obtain the stationary condition:

$$
R_{\xi \eta}(0,0):=E\left[y_{\xi 0}, y_{\eta 0}\right]=\frac{1}{\xi+\eta} .
$$

Lemma 8.2. (Determination of $\mu_{\nu}(\xi)$ ) The process y defined by (3.2) is a fractional stationary noise of order $\nu(1 / 2<\nu<1)$ if and only if:

$$
\mu_{\nu}(\xi)=\frac{\sin (\pi \nu)}{\pi}\left(\frac{2 \pi}{\xi}\right)^{\nu}
$$

Proof. From stochastic integration, we obtain from (3.2):

$$
y_{\xi}(t)=y_{\xi 0} e^{-\xi t}+\int_{0}^{t} e^{-\xi(t-s)} d \beta(s) .
$$

Then:

$$
\begin{aligned}
y(t) & =\int_{0}^{\infty} y_{\xi}(t) \mu_{\nu}(\xi) d \xi \\
& =\int_{0}^{\infty} y_{\xi 0} e^{-\xi t} \mu_{\nu}(\xi) d \xi+\int_{0}^{t}\left(\int_{0}^{\infty} e^{-\xi(t-s)} \mu_{\nu}(\xi) d \xi\right) d \beta(s) .
\end{aligned}
$$


According to the well-known formulas, when $\tau>0$ :

$$
0<\nu<1, \quad \int_{0}^{\infty} \xi^{-\nu} \exp (-\xi \tau) d \xi=\frac{\Gamma(1-\nu)}{\tau^{1-\nu}} \text { and } \frac{1}{\Gamma(\nu) \cdot \Gamma(1-\nu)}=\frac{\sin (\pi \nu)}{\pi}
$$

it is verified that, with $\mu_{\nu}(\xi)=\frac{\sin (\pi \nu)}{\pi}\left(\frac{2 \pi}{\xi}\right)^{\nu}$ and $t>s$ :

$$
\int_{0}^{\infty} e^{-\xi(t-s)} \mu_{\nu}(\xi) d \xi=\frac{(2 \pi)^{\nu}}{\Gamma(\nu)} \frac{1}{(t-s)^{1-\nu}}
$$

and

$$
\int_{0}^{t}\left(\int_{0}^{\infty} e^{-\xi(t-s)} \mu_{\nu}(\xi) \cdot d \xi\right) d \beta(s)=\frac{(2 \pi)^{\nu}}{\Gamma(\nu)} \int_{0}^{t} \frac{d \beta(s)}{(t-s)^{1-\nu}}
$$

So, for $1 / 2<\nu<1$, the noise generated by (3.2) and the fractional brownian motion (2.3) asymptoticaly behaves identically in the increment sense (for the proof, see [6] and [7]).

LEMMA 8.3. (Covariance function of $y(t)$ ). The covariance function of the $1 / f^{2 \nu}$-noise $y(t)$ is given by:

$$
R_{y}(t, s)=\int_{0}^{\infty} \varphi_{\nu}(\xi) R_{\xi \xi}(t, s) d \xi, \quad \varphi_{\nu}(\eta)=4\left(\frac{2 \pi}{\xi}\right)^{2 \nu-1} \sin (\pi \nu)
$$

Proof. For $s>t \in \mathbf{R}^{+}$,

$$
\begin{gathered}
E(y(t) y(s))=R_{y}(t, s)=\int_{0}^{\infty} \int_{0}^{\infty} E\left(y_{\xi}(t) y_{\eta}(s)\right) \mu_{\nu}(\xi) \mu_{\nu}(\eta) d \xi d \eta \\
R_{y}(t, s)=(2 \pi)^{\nu} \frac{\sin ^{2}(\pi \nu)}{\pi^{2}} \int_{0}^{\infty} \frac{\exp (-\eta(s-t))}{\eta^{\nu}}\left(\int_{0}^{\infty} \frac{1}{(\eta+\xi) \cdot \xi^{\nu}} d \xi\right) d \eta
\end{gathered}
$$

With the well-known formula $\int_{0}^{\infty} \frac{1}{(1+x) x^{\nu}} d x=\frac{\pi}{\sin (\pi \nu)}, 0<\nu<1$, it may be verified that:

$$
R_{y}(t, s)=4 \int_{0}^{\infty}(2 \pi / \eta)^{2 \nu-1} \sin (\pi \nu) \frac{\exp (-\eta(s-t))}{2 \eta} d \eta
$$

The covariance function of $y(t), R_{y}(t, s)$, may be expressed with the covariance function $R_{\eta \eta}(t, s)$ of the unique output $y_{\eta}$ only, which will give an important shortening in computations.

$$
R_{y}(t, s)=\int_{0}^{\infty} \varphi_{\nu}(\eta) R_{\eta \eta}(t, s) d \eta, \quad \varphi_{\nu}(\eta)=4\left(\frac{2 \pi}{\eta}\right)^{2 \nu-1} \sin (\pi \nu)
$$

LEMMA 8.4. (evaluation of the power spectral density $N_{y}(f)$ of the fractional noise). The PSD of the fractional noise $y(t)$ defined by (3.2) is:

$$
N_{y}(f)=\frac{1}{|f|^{2 \nu}}
$$

Proof. : $y(t)$ and $y_{\xi}(t)$ are a stationary random noise. In the sequel, we denote $t-s=\tau . y_{\xi}(t)$ is the output signal of a low-pass filter whose transfert function $H_{\xi}(f)$ is obtained by from Fourier transform with respect to $t$ of equation (3.2). It is obvious that :

$$
H_{\xi}(f)=\frac{1}{\xi+i 2 \pi f}
$$


Due to the linearity (3.2), the global transfert function of the system with input signal $w(t)$ and output signal $y(t)$ is given by :

$$
H(f)=\int_{0}^{\infty} H_{\xi}(f) \mu_{\nu}(\xi) d \xi=\frac{1}{(i 2 \pi f)^{\nu}} .
$$

Then the spectral density (or PSD) of $y(t)$ is:

$$
N_{y}(f)=|H(f)|^{2} N_{w}(f)=|H(f)|^{2}=\frac{1}{|f|^{2 \nu}}
$$

